\title{
Digitalisierung im Gesundheitswesen - zwischen Datenschutz und moderner Medizinversorgung
}

\author{
Bundeswirtschaftsministerin Brigitte Zypries hat beklagt, dass die Gesundheitswirtschaft zu \\ den am wenigsten digitalisierten Branchen gehört, obwohl schon im Januar 2016 ein E-Health- \\ Gesetz in Kraft getreten ist. Die Digitalisierung umfasst dabei verschiedene Bereiche: von der \\ elektronischen Patientenakte bis zur Telemedizin. Chancen werden vor allem in einer besseren \\ Vernetzung im Gesundheitssystem gesehen, aber auch darin, in unterversorgten Bereichen \\ Routinebehandlungen elektronisch zu unterstützen. Zudem sind Kosteneinsparungen durch \\ eine effizientere Versorgung möglich. Besser geschulte Patienten könnten intensiver in den \\ Behandlungsprozess eingebunden werden. Die größten Probleme werden beim Datenschutz \\ gesehen: Zum Schutz der Daten wird bereits viel getan, die Problematik muss aber weiter \\ beachtet werden.
}

Thomas Lux, Bernhard Breil

\section{Digitalisierung im Gesundheitswesen: bessere Versorgungsqualität trotz Kosteneinsparungen}

Die Gesundheitswirtschaft zählt laut Bundeswirtschaftsministerin Brigitte Zypries zu den am wenigsten digitalisierten Branchen. Trotz Einführung der elektronischen Gesundheitskarte 2011 und dem Inkrafttreten des EHealth-Gesetzes 2016 läuft die Digitalisierung des Gesundheitswesens nicht schnell genug. Mit der Förderinitiative „Medizininformatik" stellt das Bundesministerium für Bildung und Forschung (BMBF) nun 150 Mio. Euro für den Aufbau von Datenintegrationszentren bereit, um beispielsweise Patienten- und Gesundheitsdaten bundesweit besser für (Versorgungs-)Forschung und Entwicklung nutzbar zu machen sowie Standards für den Datenaustausch zu entwickeln und zu etablieren. Eine sektorübergreifende interdisziplinäre Kommunikation von Gesundheitsdaten wird dabei als wesentliches Element einer modernen Medizinversorgung gesehen. Andere befürchten eine Gefährdung des Datenschutzes, aus dem eine Ungleichbehandlung von Patienten resultieren könnte.

Der folgende Beitrag gibt einen Einblick in das Spannungsfeld zwischen Chancen und Risiken eines digitalisierten Gesundheitswesens und den damit erhofften Kos- teneinsparungen zugunsten einer besseren Versorgungsqualität.

Welche Bereiche sollten digitalisiert werden?

Die Digitalisierung ist ein Trend, der sich durch sämtliche Branchen zieht und zunehmend als wichtiger Erfolgsfaktor gesehen wird, insbesondere vor dem Hintergrund immer kürzerer Innovationszyklen. Stand hier vor kurzem das Informationsmanagement noch im Mittelpunkt, gewinnt derzeit die Rolle des Chief Digital Officers (CDO) in Unternehmen und Verwaltung an Bedeutung. Auch das Gesundheitswesen bleibt hiervon nicht unberührt. Dennoch ist die Frage zu klären, welche Bereiche sinnvollerweise zu digitalisieren sind. Bei der Digitalisierung sollten daher nicht die Technik, sondern die potenziellen Nutzer und deren Geschäftsprozesse im Vordergrund stehen. Die Digitalisierung ist dort sinnvoll, wo ein großer Nutzen für die Anwender ersichtlich ist und Aufwand (und somit Kosten) der Umsetzung sich in Grenzen halten. Dies setzt voraus, dass die Anwender einbezogen werden, die vielfach außen vor sind. Längst muss man im Gesundheits- 
wesen neben den professionellen Heilberufen auch Patienten und Bürger als Nutzer berücksichtigen.

Aus ärztlicher Sicht sind hier vor allem die Anwendungen der Telemedizin zu nennen, für die mit dem E-Health-Gesetz schon erste Wege geebnet wurden. Die Telekonsultation kann das Einholen einer zweiten Meinung deutlich vereinfachen und die Online-Sprechstunden helfen, gerade im ländlichen Raum, weite und unnötige Wege für Arzt oder Patient zu sparen. Beide Konzepte benötigen nicht zwingend die voll ausgebaute Telematikinfrastruktur, sondern setzen einen sicheren Austausch strukturierter Informationen in Dokumenten voraus.

Beim Blick auf die Patienten bzw. Bürger gehören die Online-Terminvergabe (die vielfach schon umgesetzt ist) sowie ein elektronischer Medikationsplan und Notfalldaten zu den tiefhängenden Früchten. Ein wesentlicher Bereich des Gesundheitswesens, der sinnvoll, wenn auch nicht vollständig, digitalisiert werden sollte, ist die Aus- und Weiterbildung. Hier ist die Digitalisierung nicht nur als Inhalt, sondern auch als Methode von großer Bedeutung. Eine Umstellung von eLearning oder Blended-LearningFormaten hat das Potenzial, dem Fachkräftemangel vor allem an der Schnittstelle zwischen Mensch und Technik entgegenzuwirken und durch entsprechende digitale Aus- und Weiterbildungsangebote somit als Multiplikator in einer globalen Digitalisierungsstrategie zu wirken. ${ }^{1}$

Neben der Betrachtung der Nutzergruppen muss die Frage nach den Geschäftsmodellen bei der Digitalisierung beantwortet werden, um einen Nutzen auch langfristig über eine Pilot- bzw. Projektphase hinaus zu gewährleisten. Viele Smartphone-Nutzer sind durchaus bereit für eine gute App Geld zu bezahlen, während andere auf werbefinanzierte Angebote setzen oder im Gegenzug mit ihren Daten bezahlen. Im Sinne einer Digitalisierungsstrategie, die einen einrichtungsübergreifenden Datenaustausch und die damit einhergehende Nutzung anstrebt, muss man sich daher die Frage stellen, welchen Stellenwert die eigenen Daten haben. Als Ziel medizinischer Informationssysteme lässt sich definieren, die richtigen Informationen zum richtigen Zeitpunkt den richtigen (= berechtigten) Personen am richtigen Ort (= überall im Krankenhaus) in der richtigen Form verfügbar zu machen. Im aktuellen Kontext muss man daher eher die Frage stellen, wo, von wem und zu welchem Zweck werden meine Daten im digitalen Gesundheitswesen erhoben, genutzt und oder weitergeleitet. Diese Frage sollte neben der technischen Komponente auch auf der ethischen Seite diskutiert werden.

1 B. Breil: Digitale Transformation durch eLearning, in: D. Matusiewicz C. Pittelkau, A. Elmer: Die digitale Transformation im Gesundheitswesen, Berlin 2017.
Was ist von der elektronischen Patientenakte zu halten?

Als wesentlicher Bestandteil vieler Digitalisierungsstrategien wird die elektronische Patientenakte (EPA) genannt. Bevor jedoch über deren Nutzen gesprochen werden kann, muss zunächst geklärt werden, was überhaupt unter der EPA zu verstehen ist. Ein wesentliches Problem im Zusammenhang mit der EPA ist, dass in vielen Diskussionen über unterschiedliche Dinge gesprochen wird. Für einige ist die EPA die vollständige digitalisierte Sammlung aller Patientendaten, für andere ein Zugriffs- oder Kontrollsystem, um Eigenverantwortung über die eigene Gesundheit zu erhalten und zu bewahren. Je nach verwendeter Definition sind auch der Digitalisierungsumfang und der zugehörige Standpunkt anders zu bewerten. Besser als die $\mathrm{x}$-te Definition ist eine Betrachtung nach Kriterien wie sie beispielweise Haas $^{2}$ vor einigen Jahren bereits vorgeschlagen hat. Als solche Einteilungskriterien verweist er unter anderem auf die Moderation der Akte (Arzt versus Patient), den Inhalt (Fallakte oder Kranken- oder Gesundheitsakte), den Umfang (alles oder wichtige Auszüge wie Notfalldaten) und einen Krankheitsbezug.

Die EPA ist sicherlich kein Allheilmittel. Sie kann in einem bestimmten Implementationsumfang Patienten helfen, Autonomie auszuüben. Zudem darf die EPA nicht als alleinstehende technische Plattform verstanden werden, welche die bislang papierbasierten Dokumentationen einfach digital weiterführt. Die eigentliche Wertschöpfung resultiert erst aus der Anpassung der Prozesse an die Potenziale der Digitalisierung. Das folgende Zitat von Bill Gates verdeutlicht, dass IT alleine nicht ausreicht, sondern auch Änderungen auf Prozessebene erfordert:

The first rule of any technology used in a business is that automation applied to an efficient operation will magnify the efficiency. The second is that automation applied to an inefficient operation will magnify the inefficiency.

Bezogen auf Deutschland bedeutet das, dass es nicht zwingend die nationale elektronische Patientenakte geben muss, sondern auf Basis verbindlicher Prozesse und Standards verschiedene EPA-Systeme am Markt angeboten werden, aus denen der Patient frei wählen kann. ${ }^{3}$ Die Heterogenität dieser Systeme spiegelt dabei die Bandbreite von sinnvollen Aktennutzungen wider.

2 P. Haas: Gesundheitstelematik, Heidelberg 2006.

3 Vgl. P. Haas: Elektronische Patientenakten - Einrichtungsübergreifende Elektronische Patientenakten als Basis für integrierte patientenzentrierte Behandlungsmanagement-Plattformen, Bertelsmann Stiftung, Gütersloh 2017. 


\section{Autorinnen und Autoren des Zeitgesprächs}

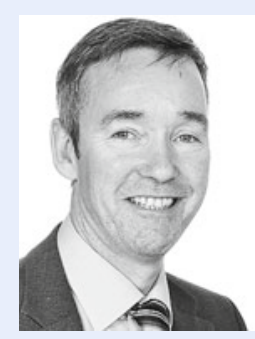

Prof. Dr. Thomas Lux ist Lehrstuhlinhaber für Prozessmanagement im Gesundheitswesen an der Hochschule Niederrhein in Krefeld.
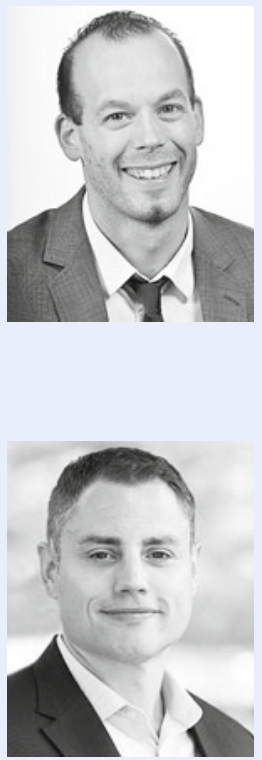

Michael Dörries, M.Sc., ist wissenschaftlicher Mitarbeiter am Lehrstuhl für Gesundheitsökonomie und Gesundheitsmanagement an der Fakultät für Gesundheitswissenschaften der Universität Bielefeld.

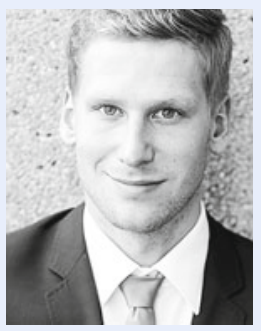

Daniel Gensorowsky, M.Sc., ist wissenschaftlicher Mitarbeiter am Lehrstuhl für Gesundheitsökonomie und Gesundheitsmanagement an der Fakultät für Gesundheitswissenschaften der Universität Bielefeld.

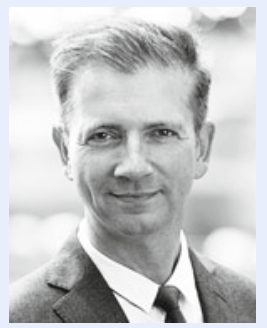

Prof. Dr. Wolfgang Greiner ist Inhaber des Lehrstuhls für Gesundheitsökonomie und Gesundheitsmanagement an der Fakultät für Gesundheitswissenschaften der Universität Bielefeld und Mitglied im Sachverständigenrat zur Begutachtung der Entwicklung im Gesundheitswesen.

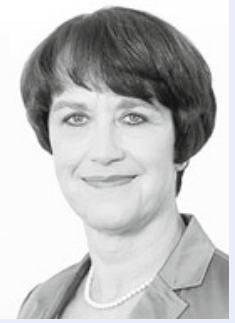

Dr. Doris Pfeiffer ist Vorstandsvorsitzende des GKV-Spitzenverbandes in Berlin.

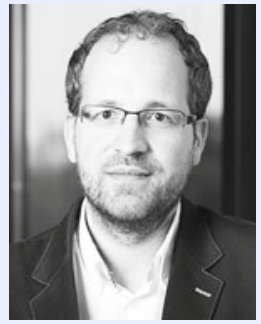

Dr. Felix G. Rebitschek ist wissenschaftlicher Mitarbeiter im HardingZentrum für Risikokompetenz am MaxPlanck-Institut für Bildungsforschung (MPIB) und Leiter des RisikoAtlasProjekts.

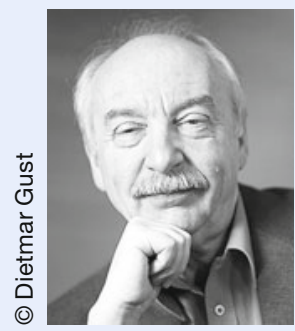

Prof. Dr. Gerd Gigerenzer ist Direktor am MPIB sowie Leiter des HardingZentrums für Risikokompetenz in Berlin.

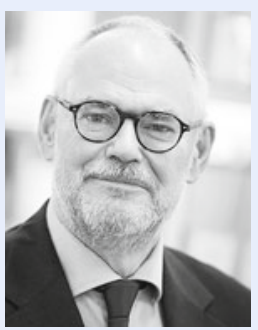

Prof. Dr. Gert G. Wagner ist Fellow am MPIB und Vorstandsmitglied des Deutschen Instituts für Wirtschaftsforschung (DIW) Berlin. 
Mehr Transparenz, Vernetzung und Kontrolle von Behandlungsmethoden und -erfolgen?

Wie in anderen Branchen (z.B. Verkehr oder Telekommunikation) ist eine passende und anforderungsgerechte Infrastruktur das wesentliche Vehikel zur Vernetzung der Akteure. Wesentlicher Aspekt im Gesundheitswesen ist, dass die Infrastruktur den Austausch ermöglicht, durch Festlegungen einheitlicher Kommunikations- und Semantikstandards. Das im E-Health-Gesetz benannte Interoperabilitätsverzeichnis ist ein Schritt in die richtige Richtung, der im Sinne der Standardisierung nicht weit genug geht. Erst verbindliche, internationale Standards schaffen eine interoperable Infrastruktur.

Ein großer Mehrwert für den Patienten entsteht gerade durch die Vernetzung verschiedener Disziplinen. Im komplexen, interdisziplinären Setting, wie es beispielsweise bei Tumorerkrankungen gegeben ist oder bei Erkrankungen, die langfristige, interdisziplinäre Behandlungen nach sich ziehen wie etwa die Mund-Kiefer-GaumenSpalte etc., kann eine Vernetzung zu mehr Transparenz und Kontrolle beitragen, nicht nur für die behandelnden Ärzte, sondern auch für den Patienten und seine Angehörigen. Es ist allerdings zu fragen, ob Ärzte und auch Patienten überhaupt einen Vorteil in mehr Kontrolle und Transparenz sehen. Auch wenn es aus der analogen Welt so naheliegend scheint, muss man sich vom reinen Dokumentenaustausch lösen, sondern vielleicht auch den Austausch einzelner Diagnosen, Datensätze im Medikationsplan oder die Ergänzung/Änderungen von Notfalldaten diskutierten. Der Dokumentenaustausch ist jedoch ein wichtiger Anfang.

\section{Effizienteres Gesundheitssystem durch Digitalisierung?}

Wie bereits angesprochen besteht eine wichtige Zielsetzung und Hoffnung in Hinblick auf die Digitalisierung im Gesundheitswesen darin, durch effizienteres Arbeiten Kosten zu sparen. Einleuchtende Beispiele für fehlende Effizienz im System sind Mehrfachuntersuchungen bei Patienten oder auch falsche Medikationen aufgrund unvollständiger Informationen mehrerer an der Diagnostik und Therapie beteiligter Akteure. Dies führt zu hohen und unnötigen direkten Kosten (z.B. mehrfache radiologische Untersuchungen, Medikationskosten) und auch zu hohen Folgekosten, beispielsweise aufgrund von Medikationsfehlern und den daraus resultierenden Folgen für den $\mathrm{Pa}-$ tienten. Bereits die digitale Vernetzung der Akteure (Ärzte) ermöglicht hier eine bessere Informationsversorgung. Darüber hinaus bestehen große Potenziale im Einsatz intelligenter Analyse- und Prognoseverfahren, die aktiv in Diagnose- und Therapieprozess eingreifen und auch bei der Steuerung klinischer Prozesse unterstützen. Dies setzt strukturierte, semantisch annotierte Daten voraus, die die Grundlage für ein effizientes Informationsmanagement bilden. Ein weiterer Mehrwert entsteht hier vor allem in der Forschung und Behandlung seltener Krankheiten, da nur durch die intelligente, einrichtungsübergreifende Vernetzung ausreichende Fallzahlen untersucht werden können. Szenarien für den Einsatz sind vielfältig, wie die Unterstützung in der Diagnostik durch Therapievorschlag (eines klinischen Behandlungspfades) anhand der Diagnoseparameter des Patienten, die Früherkennung einer Sepsis (beispielsweise derzeit forciert durch die Firma Cerner) oder auch die Verbesserung der Patientensteuerung (z.B. im Bereich des OP-Managements) zeigen.

Voraussetzung dafür ist ein deutlich höherer Grad der Digitalisierung entlang des klinischen Behandlungspfades des Patienten unter Einbeziehung sämtlicher beteiligter Subsysteme einschließlich der Patientenaufzeichnungen. Und gerade in der stationären Versorgung besteht in Deutschland noch ein hoher Nachholbedarf. Dokumentationen erfolgen oftmals papierbasiert, der Datenaustausch zwischen den beteiligten Systemen findet nur unzureichend statt und auch die Akzeptanz der Digitalisierung ist bei Ärzten, Pflegekräften und auch Patienten durchaus unterschiedlich. Erforderlich sind Investitionen in die Informationstechnologie (IT) der Krankenhäuser, in die Infrastruktur und in die Hard- und Software. Grundlegende Voraussetzung hierfür ist eine bessere personelle Ausstattung der IT-Bereiche der Krankenhäuser, die bislang vergleichsweise eher schlecht aufgestellt sind. Umdenken ist erforderlich, dass es nicht (nur) um die Verwaltung von IT-Systemen geht, sondern dass die IT ihren Beitrag zur Wertschöpfung leistet und damit zum Unternehmenserfolg beiträgt.

Hält man sich diese beiden Seiten der Medaille vor Augen, wird deutlich, dass Digitalisierung im Gesundheitswesen durchaus Kosteneinsparung ermöglicht, auf der anderen Seite aber zur Erhöhung der Kosten des IT-Betriebes führt. Auf jeden Fall aber führt die Digitalisierung zu einer Verbesserung der Versorgungs- und Behandlungsqualität des Patienten, als wichtigstes Ziel der Gesundheitsversorgung. Entsprechend sind hier Anreize in der stationären (und auch ambulanten) Versorgung für die Leistungserbringer erforderlich, um höhere Investitionen in ihre IT zu wagen.

Wenn die Kosten der Gesundheitsversorgung, die zweifelsohne im europäischen und internationalen Vergleich relativ hoch sind, diskutiert werden, sollte stets auch die gesundheitswirtschaftliche Betrachtung nicht fehlen, wie der Blick auf Beschäftigung, Wachstum, Export und weitere ökonomische Variablen. Der Anteil der Gesund- 
heitswirtschaft am Bruttoinlandsprodukt (BIP) lag 2016 bei $12 \%$ (336,4 Mrd. Euro) und die Branche beschäftigt 7 Mio. Erwerbstätige, d.h. fast jeder sechste Arbeitnehmer ist in der Gesundheitsbranche beschäftigt. Selbst in konjunkturschwachen Jahren, wie 2009 in der Finanzkrise, verzeichnet die Branche ein positives Wachstum. Im Durchschnitt liegt ihr Wachstum etwa 1,1\% über dem der Gesamtwirtschaft. ${ }^{4}$ Bereits diese wenigen gesundheitswirtschaftlichen Zahlen verdeutlichen, dass Gesundheit natürlich ihren Preis hat. Viel wichtiger ist allerdings die Betrachtung des Wertbeitrages in Form von Wertschöpfung und Beschäftigung. Allerdings ist der Wertbeitrag von E-Health mit 3,2\% (2015) im erweiterten Bereich der Gesundheitswirtschaft eher gering, was die oben getroffene Aussage der fehlenden Investitionen in IT noch stützt. $^{5}$

Wäre die Digitalisierung auch ein Mittel, um die Unterversorgung ländlicher Räume zu mildern?

Die Digitalisierung bietet vielfältige Möglichkeiten, die Versorgung von morgen zu verändern. ${ }^{6}$ Mobile Anwendungen (Apps) bieten neue und oftmals unkomplizierte Möglichkeiten, den Informations- und Datenaustausch zwischen Patient und Arzt oder auch zwischen den Leistungserbringern zu verbessern. Neben den vielen, oftmals kostenlos verfügbaren Apps, begehen auch kostenpflichtige Anbieter diesen Weg. Beispielsweise bietet die CompuGroup Medical als führender Anbieter von ArztPraxis-Lösungen für Patienten verschiedene "LifeApps“ an, die beispielsweise den Abruf ihres individuellen Medikationsplans ermöglichen oder die Kommunikation mit ihrem Arzt verbessern. Bei medatixx soll der AppPoint ${ }^{7}$ den Datenaustausch zwischen Patienten und Arzt(-praxis) durch Gesundheits-Apps ermöglichen. Auch in ländlichen, strukturschwachen Gebieten, die oftmals durch eine eher schlechte ärztliche Versorgung gekennzeichnet sind, ermöglichen digitale Lösungen verbesserte Kommunikationsmöglichkeiten. Eine wichtige Weichenstellung gegen die Unterversorgung im ländlichen Raum wurde bereits mit dem E-Health-Gesetz gelegt, welches das Telekonsil (Videosprechstunde) als Aufnahme in den Katalog der ärztlichen Leistungen festlegte. Bereits ab April 2017 können niedergelassene Ärzte diese in Form eines Technikzuschlages abrechnen und somit ihren „entfern-

4 Bundesministerium für Wirtschaft und Energie (BMWi): Gesundheitswirtschaft, Zahlen und Fakten, Ausgabe 2016, Berlin 2017.

5 K.-D. Henke: Der gesellschaftliche Nutzen der Gesundheitswirtschaft am Beispiel von E-Health, in: S. Müller-Mielitz, T. Lux: E-Health-Ökonomie, Wiesbaden 2017, S. 23-33.

6 T. Lux: E-Health - Begriff und Abgrenzung, in: S. Müller-Mielitz, T. Lux, a.a.O., S. 3-22.

7 Vgl. medatixx, https://apppoint.medatixx.de/ (10.10.2017). ten“ Patienten eine unkompliziertere Nachbehandlung ermöglichen.

Hemmschuh telemedizinischer Leistungen in der ambulanten Versorgung ist nach wie vor das Fernbehandlungsverbot ( $\$ 7$ Abs. 4 der Musterberufsordnung für Ärzte), das eine ausschließliche Beratung und Behandlung über Print- und Kommunikationsmedien ausschließt und damit den direkten Arzt-Patienten-Kontakt an einem oder mehreren Zeitpunkten erfordert. Auch würde die Aufnahme weiterer „E-Health-Leistungen“ in die Regelversorgung dazu führen, die Entwicklung voranzutreiben.

Erfolgreiche telemedizinische Versorgungsmodelle existieren bereits. So versorgt das Klinikum Oldenburg Mitarbeiter in Offshore-Windparks und auch weiteren Regionen ohne ärztliche Akutversorgung durch telemedizinische Leistungen. ${ }^{8}$ Diese und ähnliche Modelle könnten Bewohnern strukturschwacher Gegenden eine bessere Akutversorgung ermöglichen. In Baden-Württemberg hat die Landesärztekammer den Startschuss für ein Fernbehandlungs-Modellprojekt gegeben, das die ausschließliche telemedizinische Behandlung ermöglicht. ${ }^{9}$ Diese und weitere Beispiele zeigen, dass derzeit Veränderungen anstehen, die flächendeckende Versorgung zu verbessern. Allerdings sind es hier nicht die technischen Möglichkeiten, die einschränken, sondern vielmehr gilt es einen geeigneten Konsens zwischen den unterschiedlichen Akteuren zu finden, und auch für die Leistungserbringer geeignete Anreize in Form abrechenbarer Leistungen zu schaffen.

\section{Welche Gefahren sind mit der Digitalisierung verbunden?}

Neben den vielen Chancen der Digitalisierung im Gesundheitswesen gibt es auch unterschiedliche Bedenken und Risiken, die mit der Digitalisierung einhergehen (könnten). Grundsätzlich ist zu berücksichtigen, dass (patientenbezogene oder -beziehbare) Gesundheitsdaten als besondere Art personenbezogener Daten im Sinne des Datenschutzes gelten und daher entsprechend schützenswert sind ( $\$ 3$ Abs. 9 Bundesdatenschutzgesetz). Dementsprechend komplex und hoch sind die Anforderungen an die Verarbeitung dieser Daten und den Betrieb der IT-Systeme, dass deren Einhaltung den Nutzen und die Nutzbarkeit der möglichen Anwendungen stark einschränkt oder sogar verhindert. Darüber hinaus können noch weitere spezielle Regelungen, wie das Me-

8 Vgl. Klinikum Oldenburg: Telemedizin, https://telemedizin.klinikumoldenburg.de/ (10.10.2017).

9 Vgl. Landesärztekammer Baden-Württemberg, http://www.aerztekammer-bw.de/ (10.10.2017). 
dizinproduktegesetz, zu Einschränkungen bei der Entwicklung innovativer Produkte und deren Marktchancen führen. In der langjährigen Diskussion um die elektronische Gesundheitskarte (eGK) standen häufig Bedenken des Datenschutzes und der Datensicherheit im Mittelpunkt der facettenreichen öffentlichen Diskussion und trugen zunächst zu deren Verhinderung bzw. Reduktion auf nur wenige zusätzliche Funktionen bei. Allerdings ist die Erfüllung der datenschutzrechtlichen Anforderungen aus IT-Sicht durchaus lösbar, wie bereits in vielen anderen Branchen und Anwendungsbereichen bewiesen.

Im Mittelpunkt der Chancen und Risiken sollte vielmehr der eigentliche Adressat - der Patient - stehen. Wie gut ist der ländliche Bereich - und gerade der einzelne Bürger - an die digitale Infrastruktur angebunden? Welche besonderen Anforderungen und Nutzungsverhalten haben die unterschiedlicher Generationen, gerade auch die „Generation Y“ oder "Generation Z“, für die die Nutzung des Internets und mobiler Endgeräte noch selbstverständlicher ist? Auch müssen sich die Leistungsanbieter die Frage gefallen lassen, ob sich durch den Einsatz telemedizinischer (digitaler) Lösungen Diagnostik und Therapie verändern könnten. Bislang existieren hier kaum Untersuchungen, welche die Auswirkungen auf diese Berufsgruppe, ihre Arbeitsprozesse und ihr Leistungsangebot/verhalten betrachten.

Warum ist die Digitalisierung nicht weiter fortgeschritten?

Die Digitalisierung bei der Leistungserstellung ist ein Trend in allen Branchen, und auch deren Nutzung durch die Bürger. Mitarbeiter in Call-Centern werden durch Chatbots ersetzt, Banken haben den überwiegenden Teil der Kundenprozesse digitalisiert, der Friseur um die Ecke bietet elek- tronisches Terminmanagement und virtuelle Frisurenberatung an. Auch im Gesundheitswesen ist die Digitalisierung weit fortgeschritten, aber hier häufig nur direkt im Rahmen der medizinischen Leistungserstellung: Operationsroboter oder voll-digitalisierte OPs sind Beispiele hochtechnisierter Anwendungen. Andere Bereiche, wie die akteursübergreifende digitale Unterstützung bzw. Dokumentation des Behandlungsprozesses, die Vernetzung der Akteure oder der Einsatz einheitlicher Standards befinden sich gerade einmal auf dem Weg. Erklärungsansätze hierfür sind unterschiedlich. Zunächst ist die Komplexität des Gesundheitsmarktes aufgrund von Regulierungen und Beschränkungen enorm hoch, was sich durchaus innovationshemmend auswirkt, beispielsweise in Form von Marktzugangsbarrieren. Auch fehlen (finanzielle) Anreize dafür zu digitalisieren. Weiterhin ist ein Umdenken der Akteure erforderlich, nicht nur das punktuelle Ergebnis ihrer medizinischen Leistung zu sehen, sondern die Effizienz und Qualität des gesamten Behandlungsprozesses aus Sicht des Patienten.

\section{Fazit}

Die Digitalisierung ist wichtig und unaufhaltsam. Andere Branchen zeigen seit einiger Zeit das enorme Potenzial, das Digitalisierung bringen kann, wenn diese sich am Anwender und an den Prozessen orientiert. Vergleichsweise gering ist die prozessübergreifende Digitalisierung im Gesundheitswesen und die Effizienzpotenziale, die es zu heben gilt, sind entsprechend hoch. Bei allem technischen Fortschritt bleibt der Datenschutz unerlässlich für das Vertrauen der Bürger in ein digitales Gesundheitswesen und muss zwingend beachtet werden - er darf aber nicht zum Bremsklotz werden, der vor allem chronisch Kranken und multimorbiden Patienten ein Instrument der Patientensouveränität vorenthält.

\section{Digitalisierung im Gesundheitswesen - hochwertige und effizientere Versorgung}

Digitale Informations- und Kommunikationstechnologien (IKT) durchdringen heutzutage nahezu alle Lebensbereiche. Es wundert daher nicht, dass die Digitalisierung auch auf der gesundheitspolitischen Agenda zu den TopThemen zählt und intensiv diskutiert wird. Einerseits bietet der Einsatz von IKT und die digitale Vernetzung der Akteure im Gesundheitswesen große Chancen in Hinblick auf die nachhaltige Sicherstellung einer qualitativ hochwertigen und effizienten Gesundheitsversorgung. Andererseits bleiben der Ausbau einer leistungsfähigen digitalen Infrastruktur sowie die flächendeckende Etablierung von E-Health-Anwendungen in Deutschland bislang hinter den Erwartungen zurück. Eine aktuelle Studie im Auftrag des Bundesministeriums für Wirtschaft und Energie 
Abbildung 1

Potenziale der Digitalisierung

Potenziale der Digitalisierung

Leistungserbringer

- Beschleunigte Prozesse

- Genauere Therapiemöglichkeiten

- Höhere Versorgungsqualität

- Sektorenübergreifende Vernetzung

- Effizienterer Ressourceneinsatz

\section{Patient}

- Patient Empowerment

- Verbesserte Compliance

- Verbesserte Versorgung und höhere Lebensqualität

- Verkürzte Wege- und Wartezeiten
Gesellschaft/Kostenträger

- Vermeidung regionaler und fachlicher Unterversorgung

- Vermeidung von Doppelbehandlungen

- Verbesserte Behandlungsergebnisse

- Verbesserte Kosten-Nutzen-Relation

Quelle: eigene Darstellung.

(BMWi) verdeutlicht diesen Rückstand. Demnach ist das deutsche Gesundheitswesen „niedrig digitalisiert“ und im Branchenvergleich gar das Schlusslicht in Sachen Digitalisierung. ${ }^{1}$

\section{Potenziale der Digitalisierung}

Obwohl bislang digitale Strukturen in der deutschen Versorgungspraxis eher schleppend implementiert werden, bleiben die Erwartungen an den Einsatz von IKT im Gesundheitswesen ungebremst. Nutzenpotenziale lassen sich dabei auf allen Seiten, sowohl bei den Leistungserbringern und Patienten als auch auf gesellschaftlicher bzw. Kostenträgerebene, verorten (vgl. Abbildung 1). ${ }^{2}$

Für die Leistungserbringer ergeben sich zum einen Chancen zur Erschließung von Wirtschaftlichkeitsreserven, etwa durch effizientere sektorenübergreifende Kommunikationsstrukturen und beschleunigte administrative Prozesse. Die Einbindung digitaler Technologien in den Versorgungsprozess zielt zum anderen aber auch klar darauf $a b$, die Versorgungsqualität zu steigern. In die IT-Systeme der Leistungserbringer eingebundene klinische Entscheidungsunterstützungssysteme sind nur ein Beispiel dafür, wie digitale IKT an vielen Stellen im Versorgungsprozess positiv auf die Versorgungsqualität einwirken können. Sie ermöglichen Ärzten unter anderem eine schnellere und sicherere Diagnosestellung, die Auswahl sinnvoller Therapieansätze unter direkter Berücksichtigung der wissen-

1 Vgl. Kantar TNS: Monitoring-Report Wirtschaft Digital 2017 - Kompakt, Studie im Auftrag des Bundesministeriums für Wirtschaft und Energie (BMWi), Berlin 2017.

2 Vgl. R. Dittmar, W. A. Wohlgemuth, E. Nagel: Potenziale und Barrieren der Telemedizin in der Regelversorgung, in: $\mathrm{G}+\mathrm{G}$ Wissenschaft, 9. Jg. (2009), H. 4, S. 16-26. schaftlichen Evidenz und komplexer individueller Patientencharakteristika sowie die Vermeidung von Fehlern, etwa durch Interaktionswarnungen im Medikationsprozess.

Qualitätssteigerungen bei der Versorgung kommen in erster Linie dem Patienten zugute und äußern sich im Optimalfall in einem besseren Gesundheitszustand und einer höheren Lebensqualität. Darüber hinaus profitieren die Patienten aus der Einsparung von Wege- und Wartezeiten, wenn kleinere Anliegen nicht bei einem Praxisbesuch, sondern beispielsweise im Rahmen einer Videosprechstunde geklärt werden können. Eine zentrale Chance der Digitalisierung liegt zudem im Patient Empowerment, also der stärkeren Einbindung und Partizipation der Patienten im Versorgungsprozess. Die Nutzung digitaler IKT und die direkte Vernetzung der Leistungserbringer mit ihren $\mathrm{Pa}$ tienten ermöglichen es, die Idee vom mündigen Patienten Wirklichkeit werden zu lassen. Dies ist zweifelsohne zu begrüßen, kann allerdings nur funktionieren, wenn der Patient auch zum Herrscher über seine eigenen Daten wird. Anwendungen wie eine elektronische Patientenakte können nur ausreichenden Nutzen für den Patienten entfalten, wenn dieser auch selbstbestimmt über die dort hinterlegten persönlichen Gesundheitsdaten verfügen kann. ${ }^{3}$

Für die Gesellschaft bzw. die Kostenträger der privaten und gesetzlichen Krankenversicherungen stellen die zuvor genannten Aspekte eine wichtige Rationalisierungsmöglichkeit dar. Eine industriefinanzierte Studie schätzt das monetäre Effizienzpotenzial durch die Anwendung

3 Vgl. Der Beauftragte der Bundesregierung für die Belange der Patientinnen und Patienten sowie Bevollmächtigter für Pflege: Positionspapier Digitalisierung im Gesundheitswesen, Berlin 2017. 
von digitalen IKT im Gesundheitswesen auf insgesamt bis zu $39 \mathrm{Mrd}$. Euro pro Jahr. ${ }^{4}$ Insbesondere angesichts des demografischen Wandels und der damit verbundenen Gefahr regionaler und fachlicher Unterversorgung kann die Digitalisierung einen wichtigen Beitrag zur nachhaltigen Sicherstellung einer qualitativ hochwertigen Versorgung leisten. So wird es in Zukunft mehr multimorbide und chronisch kranke Patienten geben. Gleichzeitig werden auch die Ärzte immer älter, ohne dass genügend Nachwuchs rekrutiert wird, um einen ausreichenden Ersatz sicherzustellen. Bereits heute besteht in einigen strukturschwachen Regionen die Herausforderung, ausreichend niedergelassene Ärzte anzusiedeln. Die Digitalisierung bietet eine Möglichkeit, die Auswirkungen dieser Entwicklung abzuschwächen. Dabei ist nicht vorgesehen die reale Arzt-Patienten-Beziehung zu ersetzen, vielmehr soll diese um Versorgungsansätze wie etwa die Videosprechstunde ergänzt werden.

Zusätzlich zur regionalen Unterversorgung besteht in einigen Bereichen auch ein Mangel an fachlicher Expertise. So gibt es beispielsweise nur eine geringe Zahl von ausgebildeten Infektiologen. Daher lässt sich das infektiologische Fachwissen nur in telemedizinischen Kooperationsstrukturen zeitnah in der Fläche bereitstellen. Beispielhaft kann hier das Forschungsprojekt TELnet@nrw genannt werden, bei dem sich die Unikliniken Aachen und Münster disziplinen- und sektorenübergreifend mit peripheren Krankenhäusern und Ärztenetzen mittels Televisiten austauschen und die dortige infektiologische Expertise digital zur Verfügung stellen. ${ }^{5}$

\section{Digitalisierung über Sektoren- und \\ Professionsgrenzen hinweg}

Trotz einiger Schwerpunktbereiche, die vermehrt mit dem Einsatz digitaler Anwendungen in Verbindung gebracht werden (Früherkennung, Prävention, Management chronischer Erkrankungen etc.), ist grundsätzlich festzuhalten, dass die Nutzung digitaler IKT in allen Sektoren und Fachgebieten, auf viele Arten und unter Einbindung unterschiedlicher Akteure denkbar ist. ${ }^{6}$ Entsprechend lassen sich die identifizierten Chancen und Potenziale der Digitalisierung nicht auf einzelne Sektoren, Leistungsarten oder Fachdisziplinen begrenzen. Dies gilt insbesondere vor dem Hintergrund der besonderen Potenziale netzwerkba-

4 Vgl. PricewaterhouseCoopers: Effizienzpotenziale durch eHealth. Studie im Auftrag des Bundesverbands Gesundheits-IT (bvitg e.V) und der CompuGroup Medical SE, o.O. 2017.

5 Weitere Informationen unter TELnet@NRW, http://www.telnet.nrw/ (11.10.2017).

6 Vgl. F. Leppert, W. Greiner: Die Überführung von Telemedizinischen Dienstleistungen in die Regelversorgung, in: K. Möller, W. Schultze (Hrsg.): Produktivität von Dienstleistungen, Wiesbaden 2014, S. 255270. sierter Anwendungsansätze und angesichts positiver externer Effekte, die mit einer Vernetzung der Akteure über Sektoren- und Professionsgrenzen hinweg verbunden sind. So ist nicht nur der Arzt Profiteur der Nutzung einer digitalen Anwendung, die inm den Austausch von Patienteninformationen und Medikationsdaten ermöglicht. Auch andere Ärzte, Patienten, Apotheken oder Pflegeeinrichtungen, die über die digitale Infrastruktur angebunden sind, ziehen Nutzen aus dessen Gebrauch des Systems, der daraus resultierenden effizienteren Kommunikation mit dem Arzt und möglichen indirekten Effekten wie etwa der Vermeidung von Medikationsfehlern. Mit solchen Netzwerkeffekten gehen zudem positive Skaleneffekte einher, wenn auch meist nur bis zu einer gewissen Größe des jeweiligen Netzwerks. So kann angenommen werden, dass bis zu dieser kritischen Größe der Grenznutzen des gesamten Systems mit jedem neuen Teilnehmer steigt.

Die Eingrenzung der Digitalisierung auf Teilbereiche des Gesundheitswesens kann also nicht als zielführend erachtet werden. Vielmehr sollten alle Versorgungsbereiche ganz im Sinne einer sektoren- und professionsübergreifenden Versorgung in die Digitalisierungsbemühungen eingeschlossen werden. Nur wenn Medienbrüche und Kommunikationsschranken übergreifend zwischen allen Akteuren im Gesundheitswesen abgeschafft werden, kann es gelingen, das ganze Potenzial der Digitalisierung auszuschöpfen.

\section{Ursachen für den Entwicklungsrückstand}

Die Digitalisierung des deutschen Gesundheitswesens hinkt nicht nur im Branchenvergleich, sondern auch international hinterher. Die skandinavischen und baltischen Länder sind Deutschland klar voraus und haben bereits digitale Infrastrukturen geschaffen sowie elektronische Patientenakten eingeführt. ${ }^{\text {Z Z }}$ war gibt es auch hierzulande eine große Zahl von technisch verfügbaren Lösungen, allerdings werden diese aus unterschiedlichen Gründen nicht genutzt.

In der öffentlichen Debatte werden oftmals Bedenken der Patienten hinsichtlich des Datenschutzes und technischer Voraussetzungen genannt, die zu einer mangelnden Akzeptanz führen und somit eine Ausweitung der Digitalisierung behindern. Tatsächlich reagieren gerade technikunerfahrene Patienten mit relativ großer Ablehnung auf einen vermehrten Technikeinsatz im Gesundheitswesen. ${ }^{8}$ Die Beispiele von diversen Gesundheits-Apps zeigen

7 Vgl. R. Trill, A.-L. Pohl: Internationale Perspektiven von eHealth, in: F. Fischer, A. Krämer (Hrsg.): eHealth in Deutschland, Berlin, Heidelberg 2016, S. 241-256.

8 Vgl. F. Fischer, V. Aust, A. Krämer: eHealth: Hintergrund und Begriffsbestimmung, in: F. Fischer, A. Krämer (Hrsg.), a.a.O., S. 3-23. 
jedoch eindringlich, dass die Speicherung von Gesundheitsdaten kein wesentliches Hindernis für einen großen Teil der Bevölkerung darstellt. Daher ist anzunehmen, dass die Bevölkerung in Deutschland Neuerungen gegenüber grundsätzlich nicht negativer eingestellt ist als die Menschen in anderen europäischen Ländern. Die Gründe für die verzögerte Digitalisierung sind daher in anderen Bereichen zu suchen.

So können die Verzögerungen vielmehr auf die starke Fragmentierung im deutschen Gesundheitswesen und die jeweiligen Partikularinteressen zurückgeführt werden. Das pluralistische System mit seiner Vielzahl an unterschiedlichen Stakeholdern und Zuständigkeiten erschwert die Einigung auf einheitliche und umfassende Lösungen. So beschuldigten sich Ärzteschaft und Krankenkassen bereits vor der Einführung des E-Health-Gesetzes gegenseitig, die Entwicklung einer Digitalisierungsstrategie und deren Umsetzung zu blockieren. ${ }^{9}$ Dabei steht insbesondere die Frage der Vergütung mit ihren jeweiligen Anreizwirkungen im Mittelpunkt der Diskussionen. Während die Leistungserbringer eine inadäquate Vergütung verhindern wollen, befürchten die Krankenkassen eine Ausweitung der ärztlich abrechenbaren Leistungen zulasten des Versichertenkollektivs. Abgesehen von einzelnen Leistungen wie beispielsweise der Videosprechstunde, die seit dem 1. April 2017 abgerechnet werden kann, ist es daher weiterhin problematisch, wie und in welcher Höhe die ärztliche Vergütung ausgestaltet werden soll.

Die Unsicherheit hinsichtlich der Vergütung prägt auch das Verhalten der Anbieter von digitalen Anwendungen, da bis auf wenige Ausnahmen kaum Vertragsmöglichkeiten im ersten Gesundheitsmarkt genutzt werden. Der zweite Gesundheitsmarkt spielt für die Anbieter aktuell keine große Rolle, da die Zahlungsbereitschaft der privaten Nutzer zu gering ist. Dies ist darauf zurückzuführen, dass die Kosten vieler Leistungen im Gesundheitswesen üblicherweise von der Krankenkasse übernommen werden. In einer aktuellen Studie zeigt sich darüber hinaus, dass die hohen rechtlichen Hürden und der Mangel an standardisierten Regularien die Diffusion von digitalen Gesundheitsleistungen verlangsamen. ${ }^{10}$ Insbesondere der strenge Datenschutz in Deutschland wird oftmals als Hindernis für die Entwicklung und Diffusion von digitalen Anwendungen genannt. Die für den Zugang in die gesetzliche Krankenversicherung notwendigen Studien über die Wirkungen des jeweiligen Angebotes können dadurch nicht in der erforderlichen Qualität durchgeführt werden.

9 Vgl. H. E. Krüger-Brand, F. Osterloh: Gesundheitstelematik - Anreize und Sanktionen per Gesetz, in: Deutsches Ärzteblatt, 112. Jg. (2015), H. 4.

10 Vgl. F. Leppert et al.: Stärken und Schwächen der digitalen Gesundheitswirtschaft, in: Das Gesundheitswesen (2017).
Zusätzlich wird konstatiert, dass die Zulassung komplex und aufgrund der schnellen Entwicklungszyklen bei digitalen Gesundheitslösungen rein prozessual und auch aus Kostengründen für Unternehmen nur schwer zu realisieren ist.

Des Weiteren gibt es technische Umsetzungsschwierigkeiten, die die Digitalisierung im Gesundheitswesen erschweren. So müssen bestehende Prozesse und Strukturen geändert werden, um eine effiziente digitale Patientenversorgung zu gewährleisten. Dies bedeutet bei den Leistungserbringern einen erhöhten Arbeitsaufwand, den die bestehende Belegschaft im Rahmen ihrer regulären Tätigkeiten leisten muss. Diese Mehrbelastung kann zu einer geringen Akzeptanz und zu Abneigung der Mitarbeiter gegenüber der neuen Technik führen. Darüber hinaus müssen die Informationssysteme der Leistungserbringer um neue Funktionen ergänzt werden.

Die Entwicklung digitaler Anwendungen wird bisher größtenteils dem Markt überlassen. Dieses ist ordnungspolitisch zu begrüßen, führte jedoch zur Entstehung verschiedener technischer Standards. Die mangelhafte Interoperabilität der verschiedenen Schnittstellen und Anwendungen manifestiert sich in der bisher fehlenden flächendeckenden Vernetzung aller Beteiligten. Daher ist die Festlegung gemeinsamer Standards auf nationaler Ebene Grundvoraussetzung für die erfolgreiche Ausweitung digitaler Anwendungen. Dieses hat der Gesetzgeber mittlerweile erkannt und das sogenannte E-Health-Gesetz verabschiedet.

\section{E-Health-Gesetz als Treiber der Digitalisierung}

Das E-Health-Gesetz sieht die Einführung einer einheitlichen Telematikinfrastruktur unter Berücksichtigung höchster Sicherheitsstandards vor, um Leistungserbringer untereinander zu vernetzen und die Kommunikation sektorenübergreifend zu stärken. Zusätzlich wird die Vergütung der Leistungserbringer für die Verwendung von elektronischen Arztbriefen geregelt und die Einführung der elektronischen Patientenakte als zentrales Element für die Nutzung der Infrastruktur gefördert. Durch diese Anwendung soll es den Patienten im Sinne des Empowerments ermöglicht werden, sich umfassend über seine Gesundheitsdaten zu informieren und über diese im Rahmen seiner Gesundheitsversorgung eigenverantwortlich zu verfügen.

Um die Umsetzung der im E-Health-Gesetz enthaltenen Vorhaben voranzutreiben, wurden vom Gesetzgeber Fristen eingeführt, deren Nicht-Einhaltung zu Sanktionen und Haushaltskürzungen bei den verantwortlichen Stakeholdern führen können. Während das Angebot einer vergüte- 
ten Videosprechstunde 2017 einen wichtigen Meilenstein darstellte, sind für 2018 der Anschluss aller Apotheken, Krankenhäuser und Arztpraxen an die Telematikinfrastruktur vorgesehen, bevor 2019 der Zugriff von Patienten auf ihre elektronische Patientenakte erfolgen soll.

\section{Gefahren der Nutzung}

Trotz der zahlreichen Chancen, die mit einer fortschreitenden Digitalisierung verbunden sind, dürfen auch mögliche Gefahren, die mit dieser Entwicklung einhergehen, nicht aus dem Blick verloren werden. So handelt es sich bei Gesundheitsdaten um hochsensible Daten, für die höchste Sicherheit gewährleistet sein muss. Im Kontext der elektronischen Patientenakte sollen die dezentrale Datenspeicherung und die Zustimmungspflicht des Patienten vor dem Zugriff sicherstellen, dass die in der Akte gespeicherten Daten nicht für Unbefugte zugänglich sind. Darüber hinaus ist sicherzustellen, dass aus der Digitalisierung keine schädliche Ungleichbehandlung verschiedener Patientengruppen resultiert. In diesem Zusammenhang lässt sich festhalten, dass technikaffine Nutzer sicherlich früher und schneller die Vorteile der Digitalisierung nutzen werden.

Bei vielen digitalen Anwendungen wird jedoch nur die Struktur der Leistungserbringung geändert, während die Leistung selbst bestehen bleibt. Häufig handelt es sich bei den Anwendungen von Patientenseite aus zudem lediglich um eine passive Nutzung, die kein aktives Handeln erfordert. Außerdem werden telemedizinische Anwendungen in der Regel nur ergänzend zum bestehenden Versorgungsmodus eingeführt. Eine Verschlechterung der Versorgung von Nicht-Nutzern im Vergleich zum Status quo tritt daher nicht ein. Somit bietet die Digitalisierung die Möglichkeit einer Pareto-Verbesserung, bei der die Nutzer digitaler Anwendungen bessergestellt werden und etwaige Nicht-Nutzer sich nicht verschlechtern. Dennoch sollte durch flankierende Maßnahmen zur Förderung der digitalen Gesundheitskompetenz einer Ausweitung des Digital Gaps vorgebeugt werden.

\section{Ausblick}

Die Digitalisierung wird, analog zu anderen Bereichen der Gesellschaft, auch im Gesundheitswesen zu nachhaltigen Veränderungen führen. Sie eröffnet die Chance, dringenden zukünftigen Herausforderungen durch eine qualitativ hochwertige und gleichzeitig effizientere Versorgung zu begegnen. Jüngere Entwicklungen zeigen, dass die bislang eher schleppende Entwicklung der Digitalisierung im deutschen Gesundheitswesen mehr und mehr an Fahrt aufnimmt. So hat der Gesetzgeber mit dem E-Health-Gesetz verbindliche Rahmenbedingungen geschaffen. Vor allem die Knüpfung der Ausbauschritte an klare Fristen ist angesichts des derzeitigen Entwicklungsrückstands zu begrüßen.

Die starke Fragmentierung des deutschen Gesundheitsmarktes hat sich in der Vergangenheit als maßgebliches Hindernis für die Digitalisierung erwiesen. Eine Chance für ein schnelleres Voranschreiten in der Zukunft liegt daher auch in der dezentralen Entwicklung und Implementierung nutzenbringender Anwendungen durch einzelne große Akteure im Gesundheitswesen. Um die Digitalisierung im Gesundheitswesen zu beschleunigen, hat beispielsweise die Techniker Krankenkasse (TK) im Frühjahr 2017 mit dem IT-Unternehmen IBM eine Partnerschaft zur Entwicklung einer elektronischen Gesundheitsakte geschlossen.11 Die Gesundheitsakte soll für alle TK-Patienten als Satzungsleistung zur Verfügung stehen. Um Ineffizienzen durch Insellösungen und Parallelentwicklungen zu vermeiden, ist es notwendig, dass solche dezentralen Projekte gängige Standards und Schnittstellen berücksichten und eine systemübergreifende Kommunikation ermöglichen. Zudem muss sichergestellt sein, dass die Entwicklung auch anderen Akteuren zugänglich gemacht wird.

Ein Kernelement für die zukünftige Entwicklung stellt der beim Gemeinsamen Bundesausschuss angesiedelte und mit jährlich 300 Mio. Euro ausgestattete Innovationsfonds dar, der die Übersetzung der Technik in die Versorgung gewährleisten soll. Der Fonds zielt auf die Förderung neuer intersektoraler Versorgungsformen und der Versorgungsforschung ab und eröffnet die Chance, innovative digitale Anwendungen in groß angelegten Projekten zu erproben und zu evaluieren.

Nur durch die Generierung stichhaltiger Evidenz und die wissenschaftlich fundierte Evaluation der Versorgungsansätze lassen sich belastbare Aussagen über die Wirkung der Anwendung und deren tatsächliche Effizienzpotenziale treffen. Während dies in der Vergangenheit im Rahmen von überwiegend kleinangelegten Pilotprojekten meist nicht möglich war, macht der Innovationsfonds Hoffnung auf Abhilfe für die Zukunft. Die so ermöglichte Identifikation effizienter und nutzenstiftender Anwendungen sollte dann eine leichtere Überführung dieser Versorgungsansätze in die Regelversorgung ermöglichen und die Digitalisierung im Gesundheitswesen vorantreiben.

11 Vgl. Techniker Krankenkasse (TK): Die elektronische Gesundheitsakte (eGA) als wichtiger Schritt in der Digitalisierung des Gesundheitssystems, https://www.tk.de/tk/digitale-gesundheit/digitalisierungim-gesundheitswesen/interview-zur-elektronischen-gesundheitsakte/938370 (1.10.2017). 


\section{Gesundheitskarte und Telemedizin - Beispiele für die Digitalisierung}

Die Gesundheitswirtschaft gehört zu den am wenigsten digitalisierten Branchen - dieser Vorwurf wird immer wieder erhoben. So pauschal trifft er allerdings nicht zu. Tatsächlich wird in der Gesundheitswirtschaft bei einer ganzen Reihe von Prozessen bereits seit langer Zeit in digitaler Form kommuniziert. So läuft das Abrechnungsverfahren zwischen den allermeisten Leistungserbringern seit vielen Jahren elektronisch ebenso wie der Datenaustausch zwischen Arbeitgebern und Krankenkassen.

An einer Stelle hat die Informationstechnologie (IT) im Gesundheitswesen bisher allerdings wirklich erheblichen Nachholbedarf: Ärzte und Krankenhäuser dokumentieren ihre Untersuchungsergebnisse noch weitestgehend nur für die eigene Nutzung, meist in Papierform. Dadurch können Versicherte, beispielsweise bei Wohnortwechsel, nicht über die Informationen zu ihren Befunden, Diagnosen, Therapien oder Vorsorgemaßnahmen verfügen. Doch gerade das zeit- und ortsunabhängige Bereitstellen von Patientendaten ist ein wichtiges Kriterium für eine hochwertige medizinische Versorgung und ein effizientes, modernes Gesundheitswesen. Abgesehen von einzelnen Inselprojekten fehlte es bisher an einer interoperablen und sicheren Vernetzung aller Beteiligten einschließlich der Versicherten. Eine ausgebaute Infrastruktur, die einen Austausch von elektronischen Dokumenten zwischen verschiedenen Einrichtungen, Disziplinen und Sektoren möglich macht, kann vor diesem Hintergrund einen Beitrag zur Steigerung von Qualität, Transparenz und Wirtschaftlichkeit der Versorgung leisten.

Aus Sicht der Beitragszahler muss eine solche Infrastruktur selbstverständlich wirtschaftlich betrieben werden - und ein wirtschaftlicher Betrieb ist nur sichergestellt, wenn ihr nicht von potenziell unsicheren Parallelnetzen Konkurrenz gemacht wird. Da genau das seit einigen Jahren droht, war eine gesetzliche Klarstellung erforderlich. Im Sozialgesetzbuch V (SGB V) wird geregelt, dass nach Verfügbarkeit der sogenannten Telematikinfrastruktur alle Anwendungen, die eine elektronische Übermittlung medizinischer Daten vorsehen und von der gesetzlichen Krankenversicherung (GKV) gefördert und finanziert werden, von der gematik als sicheres Übermittlungsverfahren zugelassen und somit in die Telematikinfrastruktur migriert werden müssen. Die gematik ist die Gesellschaft für Telematikanwendungen der Gesundheitskarte und wird von den Spitzenorganisationen des Gesundheitswesens getragen. Eine finanzielle Förderung aus Beitragsmitteln von Anwendungen in potenziell unsicheren Netzen darf also nicht fortgesetzt werden, wenn ein durch die GKV finanziertes, sicheres Netz zur Verfügung steht.

\section{Das sichere Gesundheitsnetz steht}

Und tatsächlich gibt es ein solches sicheres Netz inzwischen - zumindest den zentralen Teil der Infrastruktur, den sogenannten Backbone. Zwischen Dezember 2016 und Juli 2017 erfolgte die Testung mit der ersten verfügbaren Anwendung, dem Versichertenstammdatenmanagement (VSDM), in Teilen von Nordrhein-Westfalen und SchleswigHolstein. An der Erprobungsphase waren 500 Arzt- und Zahnarztpraxen sowie sechs Krankenhäuser beteiligt, über 1 Mio. Online-Prüfungen der Versichertenstammdaten auf Aktualität sind durchgeführt worden. Basierend auf den umfangreichen Analysen und Auswertungen der Erprobungsergebnisse hat die Gesellschafterversammlung der gematik im Juni 2017 den bundesweiten Rollout der Telematikinfrastruktur mit dem VSDM als erste OnlineAnwendung beschlossen. Das sichere Gesundheitsnetz steht damit zum Anschluss für die Leistungserbringer zur Verfügung. Wann der Rollout faktisch beginnt, liegt nun in der Verantwortung der Industrie. Denn dazu müssen teilweise neue Komponenten auf Basis der Erkenntnisse aus der Erprobung entwickelt, durch das Bundesamt für Sicherheit in der Informationsverarbeitung zertifiziert, von der gematik zugelassen und dann in ausreichender Menge von der Industrie produziert werden. Die Kosten für die Ausstattung der Arzt- und Zahnarztpraxen und den Betrieb des Anschlusses übernimmt die GKV im Rahmen bereits abgeschlossener Finanzierungsvereinbarungen. Zur Einbindung der Versicherten in diese Vernetzung haben die gesetzlichen Krankenkassen die alte Krankenversichertenkarte bereits vor Jahren gegen eine elektronische Gesundheitskarte ausgetauscht, die als Smart-Card deutlich vielfältigere Einsatzmöglichkeiten hat und den Versicherten den Zugang zum sicheren Gesundheitsnetz ermöglicht.

Zugegebenermaßen ist die erste zur Verfügung stehende Anwendung, das Versichertenstammdatenmanagement, keine medizinische Funktion und bietet daher noch keinen wesentlichen Mehrwert für die Versorgung der Versicherten. Das VSDM sorgt jedoch für mehr Wirtschaftlichkeit, womit zumindest ein Teil der erheblichen Investitionen getragen wird. Darüber hinaus hilft diese verpflichtende Anwendung dabei, die Telematikinfrastruktur schnell zu verbreiten. Als Nächstes müssen jetzt zügig die medizinischen Anwendungen folgen. Zudem sollten die Gesundheitsanwendungen Dritter rasch die Möglichkeit erhalten, 
die Telematikinfrastruktur zu nutzen. Bereits existierende Anwendungen müssen in dieses sichere Netz migriert werden. Dabei dürfen sie natürlich nicht die Sicherheit der Telematikinfrastruktur gefährden, müssen also selbst ein angemessen hohes Sicherheitsniveau erreichen.

\section{Einführung von medizinischen Anwendungen beschleunigen}

Um diese Prozesse zu beschleunigen, haben die Gesellschafter der gematik im Spätsommer 2017 die notwendigen Voraussetzungen geschaffen: Aus dem bisherigen Vergabemodell, bei dem per Ausschreibung eine aus Beitragsmitteln finanzierte Erprobung und anschließende Freigabe für den Wirkbetrieb erfolgt, wird in Zukunft ein Marktmodell werden. Konkret heißt das: Die gematik erstellt Rahmenspezifikationen und inhaltliche Vorgaben, auf deren Basis die Industrie in einem offenen Wettbewerb eigenständig die neuen Anwendungen entwickelt und in die Fläche bringt. Sie werden anhand konkreter Bedingungen, wie beispielsweise einer eigenständig durch die Anbieter durchzuführenden Erprobung im Feld, zugelassen. Die ersten Vorgaben der gematik in diesem Sinne betreffen das Notfalldatenmanagement und die elektronische Arzneimitteldokumentation. Sie werden noch 2017 veröffentlicht und schaffen so die Voraussetzungen dafür, dass diese Anwendungen bereits 2018 eingeführt werden können. Ebenfalls noch 2017 sollen die Zulassungsbedingungen für die Etablierung und Migration von Drittanbieter-Anwendungen in die Telematikinfrastruktur erarbeitet werden.

Die Königsdisziplin der Telematik ist und bleibt natürlich die elektronische Patientenakte nach § 291a SGB V. Sie ist nicht zu verwechseln mit den aktuell am Markt angebotenen, patientengeführten elektronischen Gesundheitsakten (§ 68 SGB V). Um einen wirklichen Mehrwert zu generieren, müssen Patientenakten für alle Ärzte sowie medizinischen Einrichtungen verfügbar sein, sofern die Versicherten das möchten. Im Gegensatz zur Gesundheitsakte muss die Patientenakte zudem durch den Arzt bzw. das Krankenhaus geführt werden. Darüber hinaus brauchen die Versicherten natürlich umfassende und praktikable Einsichtsrechte ebenso wie die Möglichkeit, Lese- und Schreibrechte zu vergeben und zu verwalten. Aus diesen vielfältigen Anforderungen ergibt sich ein hoher Regelungsbedarf, vor allem bezüglich der Interoperabilität. Last but not least ist die Usability einer solch komplexen Anwendung zu gewährleisten. Es wird daher unumgänglich sein, die elektronische Patientenakte stufenweise einzuführen.

\section{Telemedizin - Chancen und Risiken}

Wenn es um die Digitalisierung des Gesundheitswesens geht, spielt nicht zuletzt die Telemedizin eine immer grö-
Bere Rolle. Und tatsächlich verfügen etliche telemedizinische Angebote über Effizienzpotenziale: Zeiten können eingespart, räumliche Distanzen leicht überwunden und relevante Informationen und Expertise schneller zur Verfügung gestellt werden.

Im April 2017 wurde deshalb die Online-Videosprechstunde eingeführt. Niedergelassene Ärzte können auf freiwilliger Basis Bestandspatienten in der Videosprechstunde betreuen, eine wiederholte persönliche Vorstellung in der Arztpraxis kann dadurch entfallen. Aktuell wird die Videosprechstunde bei bestimmten Fallkonstellationen als sinnvoll angesehen. Dabei handelt es sich mehrheitlich um Kontroll- und Verlaufsuntersuchungen, z.B. bei Wunden nach einer Operation oder bei akuten, chronischen oder offenen Wunden. Außerdem sollen Ärzte per Kamera visuell Bewegungseinschränkungen beurteilen sowie Sprech-, Sprach- und Stimmprobleme überprüfen. Die für eine ärztliche Videosprechstunde notwendigen technischen Dienste finanziert die gesetzliche Krankenversicherung über eine Pauschale. Versicherte, die eine Videosprechstunde nutzen wollen, brauchen ein internetfähiges Gerät mit Kamera, Mikrofon und Tonwiedergabe. Ärzte, die Videosprechstunden anbieten wollen, bedienen sich eines Videodienstanbieters. Dieser muss über entsprechende Sicherheitsnachweise verfügen. So muss die Videosprechstunde während der gesamten Übertragung nach dem Stand der Technik Ende-zu-Ende verschlüsselt sein. Ferner ist festgelegt, dass die apparative Ausstattung der Praxis und die elektronische Datenübertragung eine angemessene Kommunikation mit den Patienten gewährleisten müssen.

Seit 2016 besteht darüber hinaus die Möglichkeit von Telekonsilen für Röntgen- und ComputertomographieAufnahmen. Dabei wird die zeitversetzte Zweitbefundung dieser Aufnahmen durch einen Konsiliararzt durch elektronischen Austausch der Aufnahmen und sonstigen, für die Befundung relevanten Patienteninformationen möglich. Wir erwarten, dass dieser ärztliche Austausch die Qualität der Erstbefundung bei besonders komplexen medizinischen Fragestellungen wesentlich erhöht.

Die Beispiele zeigen, dass mit telemedizinischen Anwendungen ein Teil der Diagnostik und Therapie in Form einer Fernbehandlung vorgenommen werden kann. Insbesondere in ländlichen oder strukturschwachen Regionen können sie die medizinische Versorgungssituation verbessern. Wichtig ist aber, auch in Zukunft eine unmittelbare medizinische Versorgung für die Patienten zu gewährleisten. Die Telemedizin darf nicht dazu führen, dass der Sicherstellungsauftrag der ambulant tätigen Ärzte unterlaufen wird. Für die gesetzliche Krankenversicherung ist von besonderer Bedeutung, dass dem Einsatz 
von Telemedizin ein nachvollziehbarer Patientennutzen oder eine verbesserte Wirtschaftlichkeit gegenübersteht.

\section{Datenschutz - Fluch oder Segen?}

Dass der Schutz gerade von sensiblen medizinischen Daten essenziell wichtig ist, steht außer Frage. Die gesetzliche Krankenversicherung verfügt über viel Erfahrung im Umgang mit Versichertendaten und sorgt seit jeher für deren bestmöglichen Schutz - ob nun auf Papier oder digital. Tatsächlich liegt die Herausforderung darin, den Datenschutz vor dem Hintergrund neuer technischer Möglichkeiten und der damit einhergehenden Digitalisierung sinnvoll weiterzuentwickeln. Wie ist das Recht auf infor- mationelle Selbstbestimmung umzusetzen? Wie kann die Autonomie der Versicherten über ihre Gesundheitsdaten z.B. beim Aufbau der elektronischen Patientenakte sinnvoll gewährleistet werden? Das sind nur einige der Fragen, denen wir uns in diesem Zusammenhang aktuell intensiv widmen.

Fest steht dabei für die gesetzliche Krankenversicherung: Das Zählen von Schritten, verbrauchten Kalorien oder Sporteinheiten darf nicht dazu führen, dass persönliche Messdaten zu risikoadjustierten Krankenkassen-Beiträgen führen. Denn eines der wichtigsten Grundprinzipien der gesetzlichen Krankenversicherung ist die solidarische, risikounabhängige Versicherung. Und dieses Prinzip darf auch durch Digitalisierungsprozesse nicht infrage gestellt werden.

Felix G. Rebitschek, Gerd Gigerenzer, Gert G. Wagner

\section{Kritische Voraussetzungen für ein digitales Gesundheitswesen in Deutschland}

Einer Fragestellung wie jener, ob das deutsche Gesundheitswesen von der „Digitalisierung“ profitiert, könnte man sich mit abstrakten Überlegungen nähern. ${ }^{1}$ Jedoch liegt nur wenig gute empirische Evidenz vor - die nicht mit Werbeversprechen und Meinungen verwechselt werden darf - und insofern wäre eine Bewertung der Digitalisierung im Gesundheitssystem vorschnell. Unser Überblick geht deswegen einen anderen Weg: Wir versuchen kritische Voraussetzungen für digitale Innovationen, die einen überwiegenden Nutzen für die Gesundheit der Bevölkerung erwarten lassen, anhand von Fallbeispielen innerhalb des gegenwärtigen Versorgungssystems zu identifizieren. Dabei stellen wir Schlüsselfragen, deren baldige Beantwortung für die nutzenbringende Entwicklung des digitalen Gesundheitswesens erforderlich ist.

1 Die Digitalisierung fällt z.B. mit parallelen Entwicklungen der Regulation (z.B. Präventionsgesetz 2016), der Infrastrukturen (z.B. flächendeckende Telematik ab 2018) und des Verhaltens der Menschen (z.B. eigene Gesundheit vermessen) zusammen. Vgl. auch G. Gigerenzer, K. Schlegel-Matthies, G. G. Wagner: Digitale Welt und Gesundheit. eHealth und mHealth - Chancen und Risiken der Digitalisierung im Gesundheitsbereich, hrsg. vom Sachverständigenrat für Verbraucherfragen beim Bundesministerium der Justiz und für Verbraucherschutz, Berlin 2016, http://www.svr-verbraucherfragen.de/wp-content/uploads/Digitale-Welt-und-Gesundheit.pdf (10.10.2017)

\section{Vernetzte Patientendaten}

Unser erstes Fallbeispiel ist die vernetzte Bereitstellung von Patientendaten. ${ }^{2}$ Der Austausch von elektronischen Patientendokumenten, wie Arztbriefen, klinischen Aufzeichnungen und Medikationsplänen, ${ }^{3}$ zwischen unterschiedlichen Einrichtungen und Experten lässt weniger Erinnerungs-, Übertragungs-, Kommunikationsfehler, ${ }^{4}$ mehr Rückmeldungen sowie mehr Transparenz und Kontrolle von Behandlungen erwarten. Die Erfahrungen mit der elektronischen Patientenkarte illustrieren jedoch bereits technische Machbarkeitsprobleme, kritische Kos-

2 Dies fasst elektronische Patientenakte und elektronisches Patientenfach zusammen.

3 Beispielsweise elektronisch assistierte Arzneimittelgabe zur Vermeidung von Fehlern bei der Medikamentengabe und Polymedikation, vgl. H. Stürzlinger, C. Hiebinger, D. Pertl, P. Traurig: Computerized Physician Order Entry - Wirksamkeit und Effizienz elektronischer Arzneimittelverordnung mit Entscheidungsunterstützungssystemen, in: GMS Health Technology Assessment, 5. Jg. (2009), S. 56-63; eMedikationsplan für 2018 im Rahmen des E-Health-Gesetzes geplant, vgl. Bundesministerium für Gesundheit: Fragen und Antworten zur elektronischen Gesundheitskarte und zum E-Health-Gesetz, http://www. bundesgesundheitsministerium.de/themen/krankenversicherung/ehealth-gesetz/faq-e-health-gesetz.html (29.9.2017).

4 Für eine Übersicht möglicher Fehlerfaktoren, vgl. S. Taylor-Adams, C. Vincent: Systems analysis of clinical incidents: the London protocol, in: Clinical Risk, 10. Jg. (2004), H. 6, S. 211-220. 
tenentwicklungen, problematische Datenschutzfragen und ein mangelndes Vertrauen der Versicherten. ${ }^{5}$

Aus Patientensicht hat die Aufnahme in die ärztliche Versorgung schon immer bedeutet, verschiedene personenbezogene Daten offenzulegen. Zwar ist eine Datenerhebung nur dann zulässig, wenn sie für Behandlung und Abrechnung erforderlich ist, Aufnahmeformulare beinhalten aber routinemäßig weit darüber hinausreichende Daten, unter anderem Religionszugehörigkeit oder auch Nationalität. ${ }^{6}$ Mit Einführung vernetzter Patientenakten, zu denen unterschiedliche Akteure im Gesundheitssystem Zugriff haben sollen, tritt die Diskussion, welche Daten erforderlich sind, wieder in den Vordergrund. Ohne nachvollziehbare Argumente wird ein solches System wohl nicht von den Versicherten mitgetragen. Zugleich ist nicht auszuschließen, dass ein im Vorhinein festgelegtes Minimum an erforderlichen Informationen, ohne potenzielle medizinische Entwicklungen zu kennen, später hilfreiche Daten ignoriert. So könnte z.B. die Nationalität Hinweise auf bestimmte Erkrankungen liefern. Wirksam einzuwilligen heißt aber auch, ${ }^{7}$ die Verarbeitungszwecke von Daten zu kennen. Heutige digitale Patientendaten zukünftig nutzbar zu machen, z.B. für wissenschaftliche Studien, erfordert nach geltender Rechtslage auf jeden Fall spezielle Aufklärung, um hier einwilligungsfähig zu sein.

Bei der Ärzteschaft können Transparenz und ein engmaschigeres Kontrollsystem, als Folge vernetzter Patientenakten, bedeuten, dass Fehler, die andernfalls unentdeckt blieben, von anderen Akteuren rechtzeitig aufgedeckt werden. Was denjenigen, der einen Fehler gemacht hat, nicht notwendigerweise erfreut. Noch mehr „defensive Medizin“, mit einer Ausweitung unnötiger Versorgung, könnte die Folge sein, denn Fehleraufdeckung, die nur auf Bestrafung setzt, fördert defensives Entscheiden, womit Ärzte ihr eigenes Haftungsrisiko auf ein Minimum reduzieren. Solche Defensivmedizin ${ }^{8}$ lässt sich beispielsweise an der Durchführung unnötiger Tests oder auch an der Gabe von Antibiotika bei leichten Infektionen beobachten, und

5 Vgl. z.B. o.V: Elektronische Gesundheitskarte offenbar vor dem Aus, in: Süddeutsche Zeitung vom 6.8.2017, http://www.sueddeutsche. de/wirtschaft/e-card-elektronische-gesundheitskarte-offenbar-vordem-aus-1.3617842 (28.9.2017); man beachte parallele Entwicklungen wie die Versichertenkarten, vgl. o.V.: Techniker Krankenkasse vernetzt elektronische Gesundheitsakte mit Krankenhäusern, in: Ärzteblatt vom 17.8.2017, https://www.aerzteblatt.de/nachrichten/77692/Techniker-Krankenkasse-vernetzt-elektronische-Gesundheitsakte-mit-Krankenhaeusern (29.9.2017).

6 Vgl. Unabhängiges Landeszentrum für Datenschutz Schleswig-Holstein: Patientendatenschutz im Krankenhaus, https://www.datenschutzzentrum.de/medizin/krankenh/patdskh.htm (28.9.2017).

7 Vgl. ebenda.

8 Vgl. G. Gigerenzer, M. Gray: Launching the century of the patient, in: G. Gigerenzer, M. Gray (Hrsg.): Better Doctors, Better Patients, Better Decisions: Envisioning Health Care 2020, Cambridge 2011, S. 3-28. 
geht zulasten der partizipativen Entscheidungsfindung mit dem Patienten und dessen Wohl. Neue digitale Mechanismen der Fehlerminimierung verlangen somit eine neue Fehler- und Sicherheitskultur, die Beinahfehler, Lapsi und Normabweichungen ${ }^{9}$ konstruktiv aufarbeitet, deren Aufdeckung als wünschenswert etabliert ${ }^{10}$ und das Eingehen von Risiken erlaubt. Das bedeutet, vernetzte Patientendaten brauchen eine Veränderung der Defensivmedizin in eine positive Fehlerkultur. ${ }^{11}$

\section{Transparente Gesundheitsinformationen}

Die digitale Verfügbarkeit und erleichterte Zugänglichkeit zu Gesundheitsinformationen ließen erwarten, dass die Patientensouveränität bei medizinischen Entscheidungen gestärkt würde, auch bislang benachteiligte Gruppen leichter beteiligt werden und alle gesundheitlich profitieren könnten. ${ }^{12}$ Dies ist jedoch nur möglich, wenn transparente Informationen nach den Standards der wissenschaftlichen Risikokommunikation zu gesundheitsbezogenen Angeboten überhaupt vorhanden, auffindbar und vor allem laienverständlich sind..$^{13}$

Die evidenzbasierte Medizin hat Standards für die Entwicklung von Gesundheitsinformationen vorgelegt, ${ }^{14}$ sodass die beste verfügbare medizinische Evidenz Ärzten, Patienten und Versicherten verständlich gemacht werden könnte; und in begrenzter Zahl sind qualitätsgesicherte Informationsangebote bereits vorhanden. ${ }^{15}$ Sie gehen jedoch in der Masse interessengeleiteter Internetinformationen unter, welche die Standards nicht erfüllen. Dazwischen findet sich zudem Direct2Consumer-Werbung. ${ }^{16}$ Der Nutzen digitaler Gesundheitsinformationen ist daher

9 Zum Verständnis von Fehlerarten, vgl. J. Reason: Understanding adverse events: Human factors, in Quality and Safety in: Health Care, 4. Jg. (1995), H. 2, S. 80-89.

10 Zum Beispiel analog zur Luftfahrt, vgl. M. Müller: Risikomanagement und Sicherheitsstrategien der Luftfahrt - ein Vorbild für die Medizin?, in: Zeitschrift für Allgemeinmedizin, 79. Jg. (2003), S. 339-344.

11 Vgl. G. Gigerenzer: Risiko, München 2013.

12 Zum Beispiel ließen sich Überdiagnosen bzw. Überbehandlungen begrenzen, wenn individuelle Gesundheitsleistungen kritisch verstanden werden, vgl. Medizinischer Dienst des Spitzenverbandes Bund der Krankenkassen: IGeL-Monitor, https://www.igel-monitor.de/ (28.9.2017).

13 Vgl. Institut für Qualität und Wirtschaftlichkeit im Gesundheitswesen, mit dem laienverständlichen Angebot, https://www.gesundheitsinformation.de/ (29.9.2017).

14 Vgl. Arbeitsgruppe GPGl: Gute Praxis Gesundheitsinformation, in: Zeitschrift für Evidenz, Fortbildung und Qualität im Gesundheitswesen, Berlin 2016, Ausgabe 110, S. 85-92.

15 Um Beispiele aufzuführen, vgl. AOK-Bundesverband: AOK-Faktenboxen, http://www.aok.de/faktenboxen (28.9.2017); Cochrane Deutschland: Cochrane Kompakt, http://www.cochrane.org/de (28.9.2017); IQWIG: Gesundheitsinformation, https://www.iqwig.de/ (10.10.2017).

16 Vgl. o.V.: PR-Ethik-Rat rügt Agentur mhoch3 wegen gefälschter Postings, in: Der Standard vom 10.9.2015, http://derstandard. at/2000021990728/PR-Ethik-Rat-ruegt-Agentur-mhoch3-wegengefaelschter-Postings (28.9.2017). an die Entwicklung und Bekanntmachung eines qualitätsgesicherten Informationsangebots gebunden.

Die Versicherten wissen kaum, wo sie verlässliche Gesundheitsinformationen auffinden können und wie man diese von interessengeleiteten Informationen und Werbeangeboten unterscheidet. Um unter den Ergebnissen typischer Internetsuchen qualitativ hochwertige Gesundheitsinformationen aufzufinden und gegebenenfalls korrekt zu deuten, sind spezifische Fertigkeiten, wie kritisches Prüfen von angebotenen Informationen und Quellen oder das Verständnis von Risikodarstellungen erforderlich. Beispielsweise kann eine in Form relativen Risikos ausgewiesene „20\%ige Risikoreduktion“ durch eine medizinische Intervention gleichzeitig bedeuten, dass nur noch 80 statt 100 von je 1000 Patienten betroffen sind, oder, dass nur noch vier statt fünf von je 1000 Patienten betroffen sind. Ein erheblicher Unterschied, der nur anhand der absoluten Risiken erkennbar ist - wenn man das denn weiß. Dass benachteiligte Gruppen wie Kinder und Jugendliche aus Haushalten mit niedrigem Bildungsstand im Vergleich zu jenen aus Haushalten hoher Bildung bereits einen deutlich schlechteren Gesundheitszustand sowie ein nachteiliges Gesundheitsverhalten haben, ${ }^{17}$ könnte somit durch nicht ausreichende Kompetenz bei der Informationssuche und -recherche im Internet verschärft werden. Bildungsinitiativen müssen die zugrundeliegenden Risiko- und Gesundheitskompetenzen steigern, ansonsten kann sich die potenzielle Partizipation benachteiligter Gruppen als illusorisch erweisen und entsprechend könnten digitale Informationsangebote nur einer privilegierten Minderheit dienlich sein. Das bedeutet: Mehr Informationen durch das Internet helfen nichts, solange die meisten Nutzer evidenzbasierte von interessengeleiteter Information nicht unterscheiden können. Wir brauchen eine „Positiv-Liste“ von verlässlichen Quellen. ${ }^{18}$

\section{Bonusprogramme}

Die vom Gesetzgeber geforderten Bonusprogramme der Krankenkassen erlauben Rabatte auf die jeweiligen Versicherungsbeiträge, die im Vorgriff auf die antizipierte Prävention von Erkrankungen und dadurch eingesparte Kosten finanziert werden. Neben der Teilnahme an „empfohlenen Früherkennungsuntersuchungen“ werden "qualitätsgesicherte Maßnahmen der Primärpräventi-

17 Vgl. T. Lampert, C. Hagen, B. Heizmann: Gesundheitliche Ungleichheit bei Kindern und Jugendlichen in Deutschland, 2010, https://www. rki.de/DE/Content/Gesundheitsmonitoring/Gesundheitsberichterstattung/GBEDownloadsB/soz_ungleichheit_kinder.pdf (28.9.2017).

18 Unsere Forderung im Gutachten vom Sachverständigenrat für Verbraucherfragen, vgl. G. Gigerenzer, K. Schlegel-Matthies, G. G. Wagner, a.a.O. 
on" bonifiziert. ${ }^{19}$ Solche Bonusprogramme haben jedoch Probleme: Sie unterlaufen informiertes Entscheiden (z.B. bei apodiktisch empfohlenen Früherkennungen), sie bonifizieren fragwürdige individuelle Gesundheitsleistungen (die keine Kassenleistungen sind, weil der potenzielle Nutzen den Schaden nicht überwiegt, aber werbewirksam sind), sie bonifizieren „normale“ Zustandswerte ${ }^{20}$ anstatt positive Entwicklungen (was auch der Werbewirksamkeit einer Krankenkasse dient) und fördern potenzielle Risikoselektion der Versicherten sowie die unwirtschaftliche Rabattierung von Beiträgen der ohnehin präventiv aktiven Versicherten.

Die Digitalisierung vergrößert nun die Spielarten der Anreizsysteme für vermeintlich positives und kostensparendes Gesundheitsverhalten. Mobile Endgeräte erlauben die Erfassung sportlicher Aktivitäten per Tracking, sodass beispielsweise ein Durchschnittspuls von mindestens 120 Schlägen pro Minute innerhalb von 30 Minuten, mindestens 10000 Schritte pro Tag oder ein durch Bewegung erreichter Mindestverbrauch von 150 Kalorien innerhalb von 30 Minuten bonifiziert werden können. ${ }^{21}$ Solche Erfassungen ersetzen mithin äußerst fragwürdige Proxies, wie die bloße Mitgliedschaft in Fitnessstudios. Theoretisch könnte diese Detailerfassung des Verhaltens von Versicherten positiv bewertet werden, indem das Erreichen von gesunden Zuständen bonifiziert würde.

Dies ist in der Realität jedoch insofern problematisch, da Normalbereiche physiologischer Zustände als vom Individuum zu erstrebende Normen und Normwerte etabliert werden, die nicht notwendigerweise gesund sind. Nicht nur gab es in der Vergangenheit wiederholte Neudefinitionen der Normalbereiche sondern auch die zugrundeliegenden Zusammenhänge mit der Gesundheit sind nicht immer vollständig verstanden und Gegenstand wechselnder Interpretationen. ${ }^{22}$ Des weiteren wird beispielsweise die Teilnahme an Früherkennungsuntersuchungen belohnt, obgleich viele Teilnehmer auch mit Schäden rechnen müssen, während zugleich keine Evidenz exis-

19 Auf Bewegung, gesunde Ernährung und Vermeidung von Suchtmittelkonsum und Stress abzielend, vgl. Verbraucherzentrale NRW: Bonusprogramme der gesetzlichen Krankenkassen - Anreiz für gesundheitsbewusstes Verhalten oder Prämienzahlung für Gesunde?, September 2015, https://www.verbraucherzentrale.nrw/sites/default/ files/migration_files/media236794A.pdf (28.9.2017).

20 Z.B. Cholesterin, Blutzucker, Blutdruck, Body-Maß-Index (BMI).

21 Vgl. AOK Plus: Bonifizierbare Aktivitäten, https://www.aokplusonline.de/fileadmin/user_upload/bonusprogramm/BonifizierbareMassnahmen-AOK-PLUS-Bonusprogramm.pdf (28.9.2017).

22 Für das Beispiel des gesunden, übergewichtigen BMls von 27.0, vgl. S. Afzal, A. Tybjærg-Hansen, G. B. Jensen, B. G. Nordestgaard: Change in body mass index associated with lowest mortality in Denmark, 1976-2013, in: Jama, 315. Ausgabe (2016), H. 18, S. 1989-1996. tiert, dass innerhalb einer langjährigen Beobachtungszeit weniger Teilnehmer als Nichtteilnehmer sterben. ${ }^{23}$

Die individuelle Gesundheit von Patienten kann weder durch „temporäre“ Normen, die letztlich einen substanziellen Mangel an Evidenz einschließen und deswegen jederzeit revidiert werden können, noch durch Normen auf Basis hochqualitativer Evidenz gefasst werden, denn bislang gibt es letztendlich keinen Beleg, dass bestimmte Aktivitäten für jeden Versicherten gleichermaßen gesund sind; und gar chronisch Kranke und ältere Menschen hierbei nicht tendenziell benachteiligt würden. Würde sich die digitale Selbstvermessung in graduellen, langfristig verteilten Stufen zu einer Art „Bürgerpflicht“ entwickeln, wäre dies auf Basis des heutigen Wissens nicht verantwortbar. Und (unverschuldet) chronisch Kranke müssten vor solch „statistischer Diskriminierung“ geschützt werden, um den Solidargedanken nicht zu zerstören. Das bedeutet: Bonusprogramme müssen strikt evidenzbasiert werden und nicht „werbewirksame“ Leistungen für fragwürdiges Gesundheitsverhalten anbieten, wie es derzeit teilweise der Fall ist.

\section{Digitale Laien- bzw. Patientenunterstützung}

Wesentliche Gefahren durch die Digitalisierung müssen vor allem in der Vielzahl ungeprüfter Angebote für Laien gesehen werden. Obwohl nicht als Medizinprodukt erfasst, entwickelte sich in den vergangenen Jahren als Konterpart zum klassischen Gesundheitssystem eine Palette von digitalen Produkten, welche die Gesundheit des Einzelnen beeinflussen kann oder soll. ${ }^{24}$ Außerhalb des Medizinprodukte-Gesetzes sind sie für eine Analyse des klassischen Gesundheitssystems relevant. Sie könnten unter Umständen als Medizinprodukte einzustufen sein und sie lassen auf jeden Fall Auswirkungen auf den Nutzer, dessen Gesundheit, die Arzt-Patienten-Interaktion sowie die allgemeine Versorgungssituation erwarten, ohne dass ausreichend qualitative Evidenz zu potenziellen Nutzen und Schäden vorliegt. ${ }^{25}$

23 Vgl. V. Prasad, J. Lenzer, D. H. Newman: Why cancer screening has never been shown to "save lives“ - and what we can do about it, in: British Medical Journal, 6.1.2016, 352, http://www.bmj.com/content/352/bmj.h6080 (10.10.2017).

24 Neben Informationswebseiten, Textnachrichten und Apps gehören dazu auch Wearables (Fitness-Armbänder, Fitness-Uhren, Ohrclips, Sensorpflaster), deren weltweites jährliches Marktvolumen im Healthcare-Bereich für 2020 auf etwa 41 Mrd. US-\$ geschätzt wird, vgl. Soreon Research: The Wearable Health Revolution, How Smart Wearables Distract the Healthcare Sector, Executive Summary of the Soreon Report, 2014, http://www.soreonresearch.com/wp-content/ uploads/2014/09/Extract-Soreon-Research-Report-The-WearableHealth-Revolution.pdf (28.9.2017).

25 U.-V. Albrecht (Hrsg.): Chancen und Risiken von Gesundheits-Apps, CHARISMHA, Hannover 2016, http://www.digibib.tu-bs.de/?docid=00060000 (29.9.2017). 
Über ihre massenhafte Verbreitung sind diese Produkte für das Gesundheitssystem als Ganzes relevant, da sie zum einen unnötige Inanspruchnahme des Versorgungssystems motivieren können, zum anderen unter Umständen ärztlich unbegleitete Interventionen anstoßen. Außerdem suggerieren viele digitale Produkte außerhalb des Medizinprodukte-Gesetzes eine Verantwortlichkeit für den eigenen Gesundheitszustand, der nicht notwendigerweise mit der medizinischen Evidenz korrespondiert, obgleich mit den Produkten unbewiesene Überzeugungen weithin verbreitet werden. Dies wird umso problematischer, wenn Solidarität an individuelle Verantwortungsübernahme gekoppelt wird, obwohl diese eben nicht immer möglich ist (z.B. durch Veranlagungen). Die Verbreitung digitaler Assistenten für die Allgemeinbevölkerung muss somit von Wirksamkeitsbelegen und gesellschaftlichen Auseinandersetzungen äußerst kritisch begleitet werden.

\section{Wie die digitalen Chancen nutzen?}

Bevor das analoge Gesundheitssystem Nutzen aus der Digitalisierung ziehen kann, müssen zuerst die Probleme beseitigt werden, die bereits seit langem im Gesundheitswesen eine Rolle spielen. Wenn dies nicht erfolgt und folgende kritische Voraussetzungen nicht erfült werden, kann die Digitalisierung die Probleme nur überdecken bzw. wird sie verschärfen. In diesem Fall sind forcierte Schritte zu flächendeckender Digitalisierung im Gesundheitswesen nicht anzuraten.

\section{Drei kritische Voraussetzungen:}

1. Wir müssen Maßnahmen ergreifen, um die erforderliche Risiko- und Gesundheitskompetenz von Versicherten sicherzustellen. Dadurch kann die gesetzlich geforderte aktive Rolle bei der eigenen Gesundheit wahrgenommen werden. Um den durch die Digitalisierung erweiterten Anforderungen und dem damit verbundenen Fehlerpotenzial bei der Informationssuche und -bewertung zu begegnen, wird eine Risikokompetenz- und Gesundheitsbildung benötigt. Dies sollte bereits in der Schule beginnen, aber auch die gegenwärtig schon erwachsene Bevölkerung erreichen. Zudem ist ein unabhängiges, qualitätsgeprüftes und auffindbares Angebot von verständlichen digitalen Gesundheitsinformationen erforderlich. Nur mit diesen Informationen ist informiertes Entscheiden von Versicherten, das durch digitale Informationsangebote grundsätzlich verbessert wird, überhaupt möglich.

2. Wir müssen die Ausweitung von Defensivmedizin stoppen. Damit die durch digitale Dienste und Geräte ermöglichten Erfassungs-, Rückmeldungs- und Kontrollmechanismen nicht dazu führen, dass Ärzte ihre Haftungsrisiken zulasten des Patientenwohls minimieren, ist Spielraum zum Eingehen der tragbaren Risiken und Organisationsformen, die aus Abweichungen von risikominimierenden Verhaltensweisen lernen, nötig. Hierbei müssen die Arbeitgeber und Selbstverwaltungseinrichtungen (wie die Ärztekammern) für die Umsetzung positiver Fehlerkulturen, die sich an der Luftfahrt orientieren können, Verantwortung übernehmen.

3. Wir sollten die Solidarität im Gesundheitswesen sichern. Um angesichts bislang ungeprüfter digitaler Werkzeuge zur Kontrolle der eigenen Gesundheit die Verantwortung des einzelnen Gesellschaftsmitglieds überhaupt diskutieren zu können, ist zu allererst der Nachweis ihrer Wirkungen nötig. Qualitätsevidenz aus randomisiert-kontrollierten Studien muss zeigen, für wen die Nutzung welcher Werkzeuge gesundheitsförderlich ist. Und wer davon nicht profitiert und geschützt werden muss. Des Weiteren sind solche Nachweise verständlich zu erklären. Erst dann ist eine gesellschaftliche und politische Auseinandersetzung darüber verantwortbar, inwieweit individuelle Verantwortung für die eigene Gesundheit über Körper und Geist ins Portemonnaie hineinreichen könnte und sollte.

Fazit: Digitalisierung im Gesundheitswesen braucht drei Voraussetzungen: risikokompetente Versicherte, weniger Defensivmedizin und Solidarität.

Title: Healthcare - Between Privacy and State-of-the-Art Medical Technology

Abstract: The digital transformation of the healthcare branch is important and unstoppable. The enormous possibility of digitalisation to redesign and enhance processes has been shown in other branches. Digital technologies offer the similarly large potential to improve the quality and efficiency of healthcare services. However, in terms of digitalisation, the German health system lags behind other European countries. But given a lack of available evidence, the effects of the digitalisation of the healthcare system cannot be reliably evaluated. Digital access to patient data necessitates constructive error cultures in organisations in order to limit defensive medicine. And digital access to health information necessitates individual competence in searching and using this information for participatory decision-making. As with all technological progress, information security is very important in order to gain citizens' confidence in a digital healthcare system. Thus, it is necessary to simultaneously pursue both enhanced privacy standards and state-of-the-art medical technology.

JEL Classification: $110,111,118$ 